\title{
Design of The SiCN Temperature Sensor Detecting System Based on LabVIEW
}

\author{
Tianli Li, Junli Xu, Gang Xu*, Donghui Zhou ${ }^{4}$ and Yuansi Chen ${ }^{5}$ \\ Shenzhen Key Laboratory of Electromagnetic Control, Shenzhen University, Shenzhen 518060, China \\ *Corresponding author
}

\begin{abstract}
The SiCN temperature sensor is a passive wireless sensor cavity, which uses SiCN ceramic as dielectric resonator, the material has some advantages such as thermal shock, corrosion and oxidation resistance, etc. Through the analysis of resonator sensor modeling, uses the theory of weak-disturbance modifying measuring, and uses the NI Company of the PXIe$5644 \mathrm{R}$ vector signal transceiver (VST), circulator, waveguide and high-frequency devices, the paper designs a SiCN temperature sensor detection system based on LabVIEW. The PC displays processing analysis and storages the sensor signal, so as to measure the characteristics of the sensor. The system can detect a variety of models of the sensor, and has a strong ability to upgrade and expand.
\end{abstract}

Keywords-the SiCN temperature sensor; perturbation method; echo signal; signal analysis

\section{INTRODUCTION}

With the development of industrial production, many industrial production environment is bad, in order to ensure the equipment is good and stable, we need to test the parameter of the environment (such as temperature, pressure, humidity, etc.). In many occasions, these parameters of the equipment have been able to meet our requirements, but for some special equipment, the working environment is more complex and severe. For example, If the working temperature reach $3000^{\circ} \mathrm{C}$, the common testing instrument can't meet our requirements. So it is very important to study a new type of temperature measurement system for the special environment of industry.

This paper uses the theory of weak-disturbance modifying measuring, and uses the NI Company of the VST, circulator, waveguide and high-frequency devices, the paper designs a SiCN temperature sensor detection system based on LabVIEW. The system has a high degree of openness, automation and practicality, it can detect the sensor quickly and automatically.

\section{MeAsuring PrinciPle OF SENSOR}

The SiCN temperature sensor is a passive wireless sensor cavity, when the physical condition of the microwave cavity have small changes, such as the temperature, humidity or medium different in the cavity, these changes affect the dielectric permittivity of the sensor cavity, which will cause the small changes in the resonant frequency, and then will cause the small changes of resonant frequency [1]. This paper uses a new type of nondestructive testing technology (microwave detection technology) to detect the sensor.
The following formula is the formula of the Sensor working principle.

$$
f=\frac{1}{2 \pi \sqrt{\mu_{0} \varepsilon_{0} \varepsilon_{r}}} \frac{x_{01}}{r}
$$

In the formula, $f$ is the resonant frequency of the resonator, $\varepsilon_{0}$ is the dielectric constant under the condition of vacuum, $\mu_{0}$ is the permeability under the condition of vacuum, ${ }^{x_{01}}$ is the roots of the first Bessel function, $\varepsilon_{r}$ is the dielectric constant, $r$ is the radius of the sensor[2].

As shown in figure 1, in this paper, the principle of the sensor is electromagnetic interference. The incident electromagnetic wave and the reflected electromagnetic wave interfere in the medium surface, thus absorb the incident electromagnetic wave energy from the outside. When the resonant frequency of the incident signal is consistent with the natural frequency of the resonant cavity medium, the absorption capacity reaches the maximum, and the power of the echo signal is the smallest[3, 4].

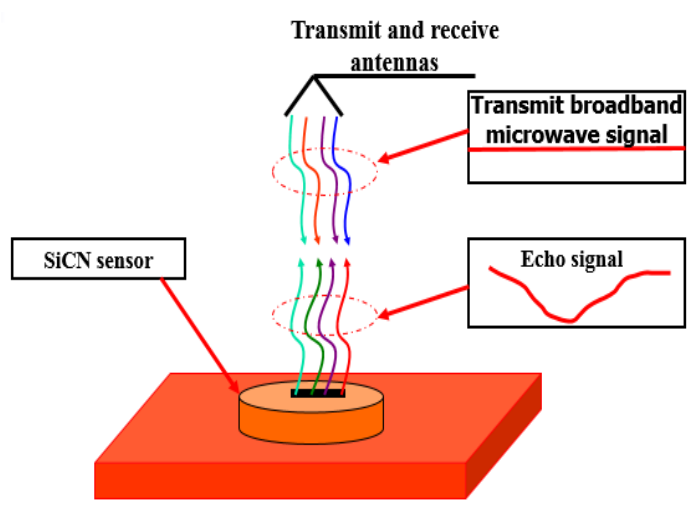

FIGURE I. WORKING PRINCIPLE DIAGRAM OF SICN SENSOR

\section{SYSTEM HARDWARE CONSTRUCTION}

As shown in figure 2, the system mainly consists of 4 modules: VST, circulator, waveguide, resonant cavity sensor. VST is the key module of the system, it mainly includes vector signal generator (VSG) and vector signal analyzer (VSA). The function of VSG is to produce the radio frequency signal, and 
the function of VSA is to receive the echo signal. The function of circulator is unidirectional transmission and reverse isolation. The Waveguide is mainly used to transmit radio frequency energy. In this paper, it is used between the sensor and the ring device. This paper use the sweep method to detect the sensor. Using LabVIEW PC to control VST to generate a frequency sweep signal, through the circulator and waveguide converter transmit RF signals to the SiCN sensor in a wireless way. After a certain period of response, PC acquires, displays processing analysis and storages the sensor signal, so as to measure the sensor's characteristics.

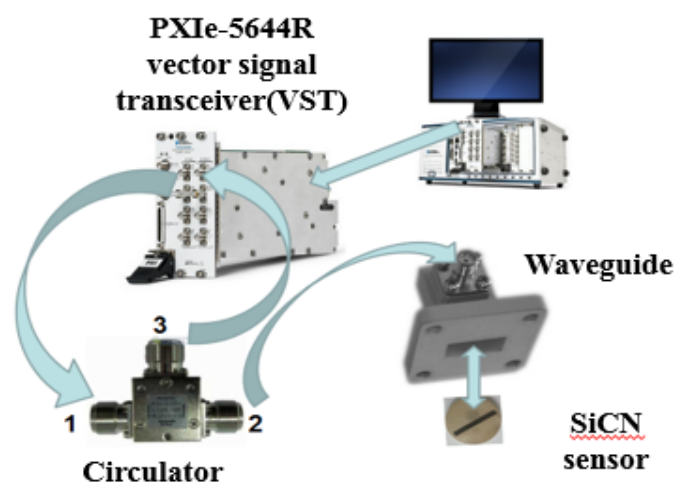

FIGURE II. SYSTEM STRUCTURE DIAGRAM

\section{SYSTEM SOFTWARE DESIGN}

This part is the core part of the system, signal transmission, signal acquisition, signal processing, analysis and display are all controlled by the software system. As shown in Figure 3, the system is mainly divided into the following modules: signal source module, signal acquisition module and processing display module $[5,6]$.

\section{A. Signal Source Module}

Signal source module is the key module of the system, this paper uses VSG as the signal generator module. The user first set the sweep frequency parameters in the front panel and then control the VSG achieve the desired objectives. This system can realize $65 \mathrm{MHz}$ to $6 \mathrm{GHz}$ frequency range, and the frequency sweep step can reach the minimum $3 \mathrm{kHz}$, the sweep time step can reach 50 us. As shown in Figure 4, it is the signal source module program.

\section{B. Signal Acquisition and Processing Module}

Signal acquisition send the echo signal which returned from the sensor to the PC for analysis and processing. This paper uses the VSG as the receiving hardware of the echo signal, and combined with the software of the PC composed the signal acquisition module.

As shown in Figure 5, the signal processing mainly collect the echo signal, choose the appropriate filtering algorithms to filter out noise in the echo signal, extract the resonant frequency, storage and display the results. As shown in Figure 5 , the signal processing is mainly to deal with the echo signal, extracting resonant frequency, storing and displaying the results.

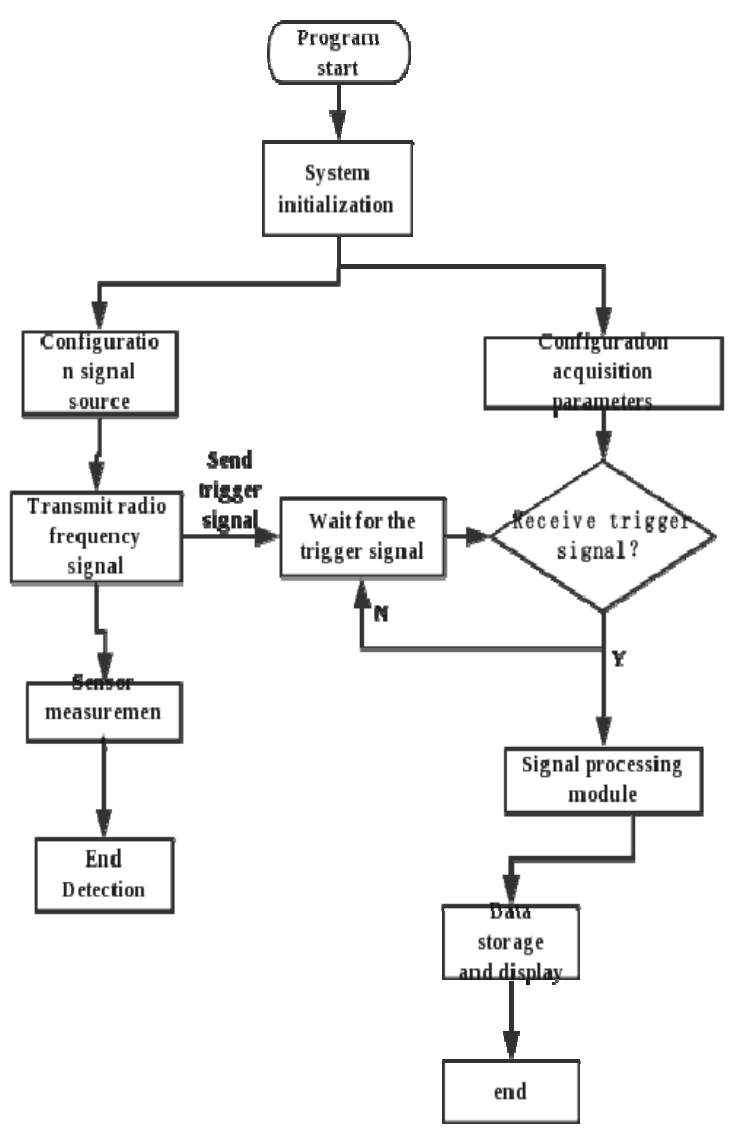

FIGURE III. MAIN FLOW CHART

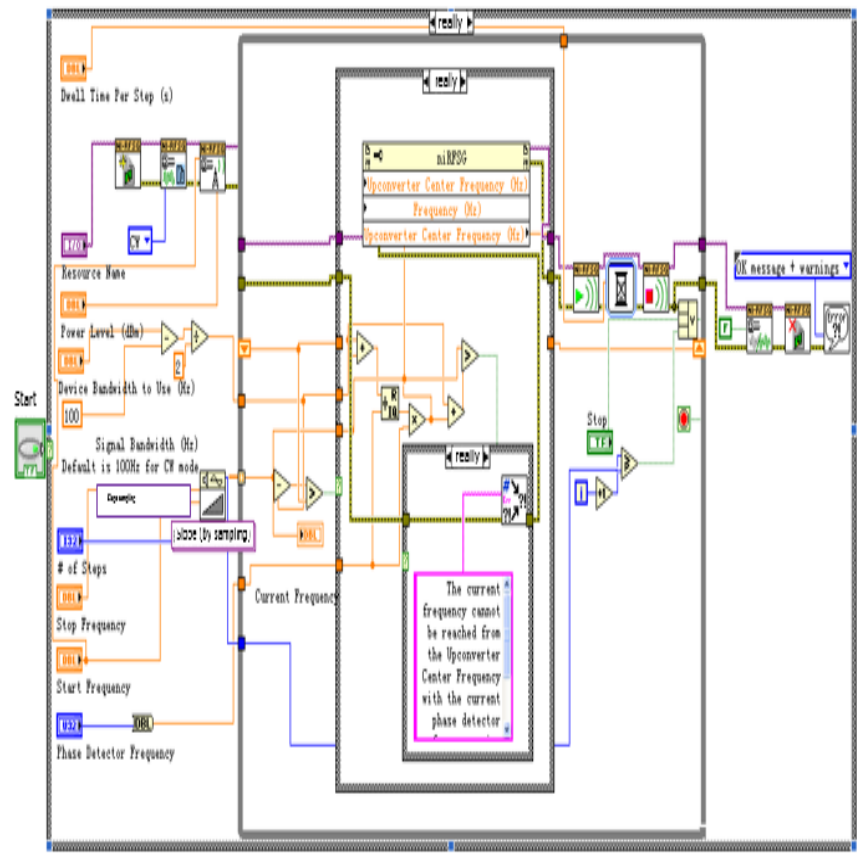

FIGURE IV. SIGNAL SOURCE MODULE PROGRAM 


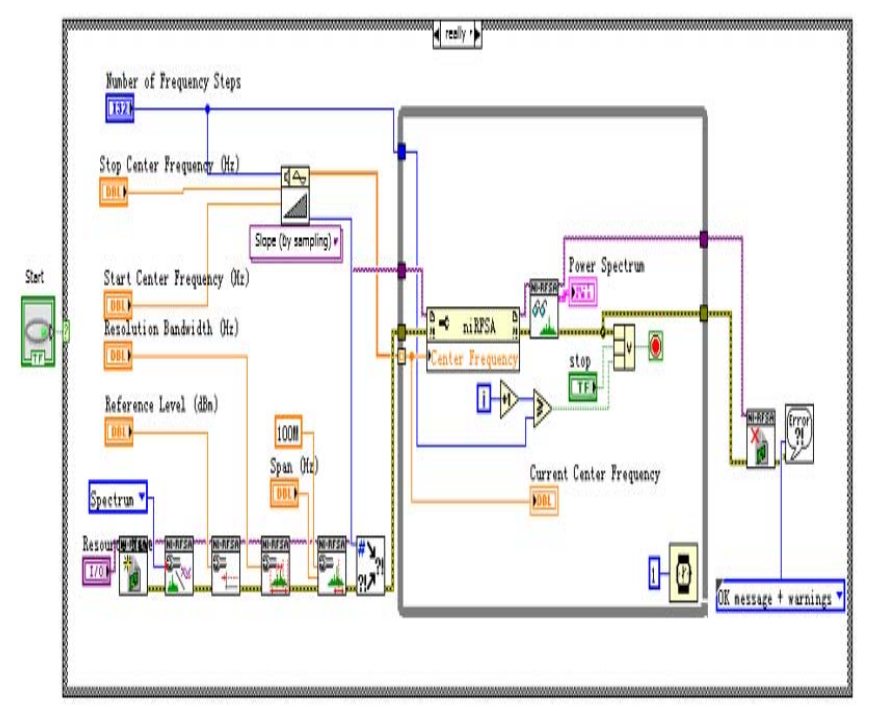

FIGURE V. SIGNAL ACQUISITION AND PROCESSING MODULE

\section{RESULTS TEST AND ANALYSIS}

Placing sensors under the waveguide, adjusting the position of sensor and waveguide, finding the position of the resonance point curve, at this time, the signal attenuation is the largest, and the coupling efficiency of the sensor and the waveguide is the highest. At the temperature of $25{ }^{\circ} \mathrm{C}$, comparing the measurement results with or without sensor, and drawing the curve of the echo signal. As shown in Figure 6, compared the curve before and after adding the sensor, it is found that there is an obvious change, and in the valley near the change is biggest, the sensor leads to the loss of energy and has obvious influence on the result.

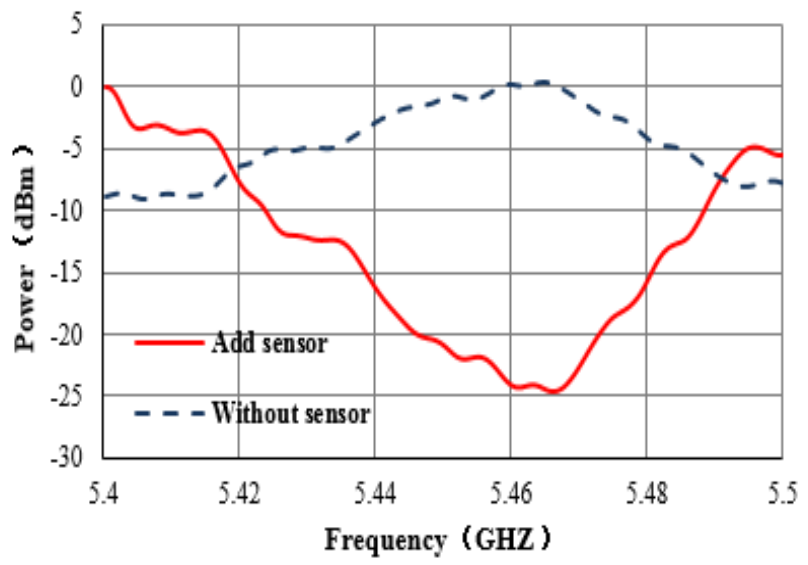

FIGURE VI. RESULT COMPARISON CHART BEFORE AND AFTER ADDING SENSOR

Heating the sensor, adjusting the position of the sensor and the waveguide, configuring sweep parameters, recoding the resonance frequency in the table I. And drawing the curve of temperature and resonance frequency. As shown in Figure 7, it can be seen that the resonant frequency of the sensor decrease with the increase of the temperature, when the temperature rises $250{ }^{\circ} \mathrm{C}$, the resonant frequency of the sensor reduce by 11.49 $\mathrm{MHz}$, fitting the actual curve, and to find the first order partial derivatives. While in the 50 to $300{ }^{\circ} \mathrm{C}$ temperature experiment conditions, the sensitivity of the sensor is about $49.96 \mathrm{KHz} /{ }^{\circ} \mathrm{C}$.

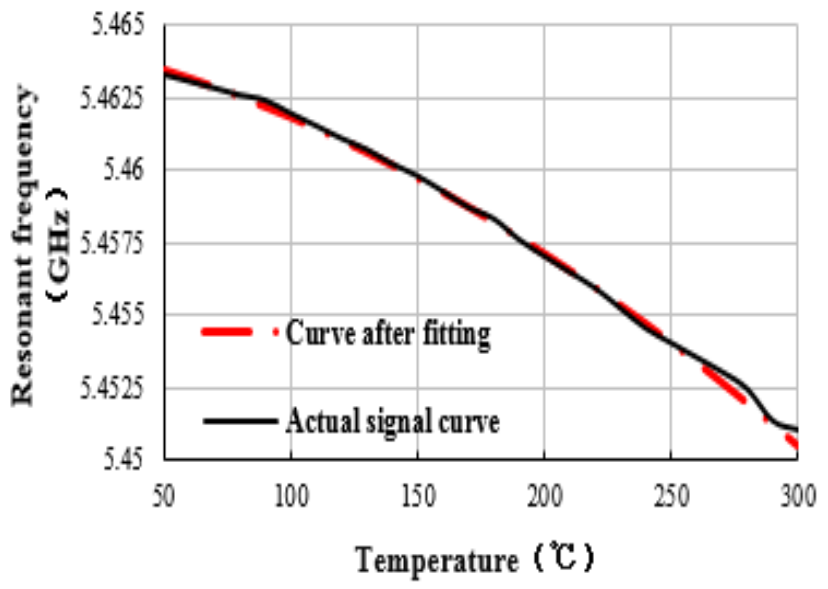

FIGURE VII. RELATIONSHIP BETWEEN RESONANCE FREQUENCY AND TEMPERATURE

\section{CONCLUSION}

This paper is based on the research of SiCN microwave resonant cavity sensor, and design of the SiCN temperature sensor detecting system based on LabVIEW. The system can be used to detect sensor's performance, after experimental verification, the system is stable, accurate measurement, data processing ability, strong practicability, and has great application prospect. Using the system to detect the SiCN sensor and calculating the resonant frequency of the sensor by the relevant algorithm. While in the 50 to $300{ }^{\circ} \mathrm{C}$ temperature experiment conditions, the sensitivity of the sensor is about $49.96 \mathrm{kHz} /{ }^{\circ} \mathrm{C}$.

\section{REFERENCES}

[1] Yu Jiang, Jun Cao, Guohui Yang. Method for Designing Moisture Sensor Based on Microwave Resonant[J]. Modern Scientific Instruments, 2006, 2.

[2] Zhiyuan Lu, Wenquan Sui, Zhigang Wu et al.A Study on Microwave Resonance Cavity Sensor for Detecting Cigarette Humidity and Density[J].Chinese Journal of Sensors and Actuators, 2007, 5.

[3] Yan Li, Yuxi Yu, Haisheng San,et al. Wireless passive polymer-derived SiCN ceramic sensor with integrated resonator/antenna[J]. Applied Physics Letters, 103, 2013, 103(103): 1-4.

[4] Jia Yan, Anjie Wang, Dong-Pyo Kim.Preparation of ordered mesoporous SiCN ceramics with large surface area and high thermal stability[J]. Microporous and Mesoporous Materials, 2006, 100(1): 128-133.

[5] Jiayong Han. Software Development for Ash Scanner Based on Virtual Instrument Technology[D]. BeiJing: Tsinghua University, 2012: 16-29.

[6] K.N.WHITLEY, ALANF. BLACKWELL. Visual Programming in the Wild:A Survey of LabVIEW Programmers [J]. Journal of Visual Languages and Computing, 2001, 12: 124. 
TABLE I. RESONANT FREQUENCY VALUES WITH TEMPERATURE VARIATION

\begin{tabular}{|c|c|c|c|c|c|}
\hline Sequence number & Actual temperature $\left({ }^{\circ} \mathrm{C}\right)$ & Resonant frequency $(\mathrm{GHz})$ & Sequence number & Actual temperature $\left({ }^{\circ} \mathrm{C}\right)$ & resonant frequency $(\mathrm{GHz})$ \\
\hline 1 & 50 & 5.46329 & 14 & 180 & 5.4583 \\
\hline 2 & 60 & 5.46306 & 15 & 190 & 5.45758 \\
\hline 3 & 70 & 5.46282 & 16 & 200 & 5.457 \\
\hline 4 & 80 & 5.46258 & 17 & 210 & 5.45645 \\
\hline 5 & 90 & 5.4624 & 18 & 220 & 5.4559 \\
\hline 6 & 100 & 5.46195 & 19 & 230 & 5.4552 \\
\hline 7 & 110 & 5.46152 & 20 & 240 & 5.454 \\
\hline 8 & 120 & 5.46108 & 21 & 260 & 5.4535 \\
\hline 9 & 130 & 5.4607 & 23 & 270 & 5.4530 \\
\hline 10 & 140 & 5.4602 & 24 & 280 & 5.4524 \\
\hline 11 & 150 & 5.45978 & 25 & 290 & 5.4513 \\
\hline 12 & 160 & 5.45915 & 5.4587 & 300 & 5.4511 \\
\hline 13 & 170 & & & & \\
\hline
\end{tabular}

